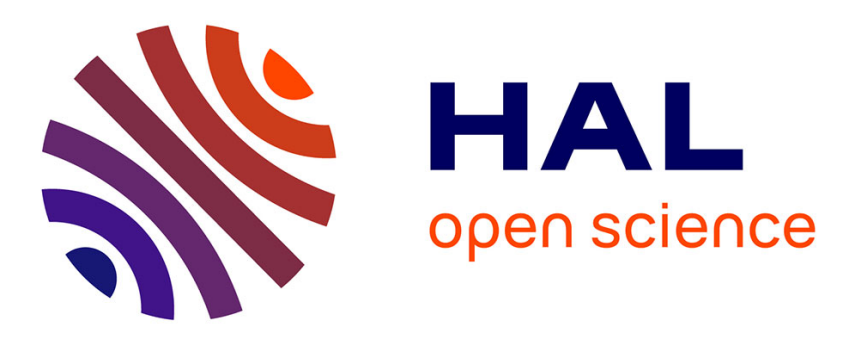

\title{
Mechanism of multiple pulse formation in the normal dispersion regime of passively mode-locked fiber ring lasers
}

Adil Haboucha, Andrey Komarov, Hervé Leblond, François Sanchez, Gilles Martel

\section{To cite this version:}

Adil Haboucha, Andrey Komarov, Hervé Leblond, François Sanchez, Gilles Martel. Mechanism of multiple pulse formation in the normal dispersion regime of passively mode-locked fiber ring lasers. Optical Fiber Technology, 2008, 14 (4), pp.262 - 267. 10.1016/j.yofte.2008.01.001 . hal-03423694

\section{HAL Id: hal-03423694 \\ https://univ-angers.hal.science/hal-03423694}

Submitted on 10 Nov 2021

HAL is a multi-disciplinary open access archive for the deposit and dissemination of scientific research documents, whether they are published or not. The documents may come from teaching and research institutions in France or abroad, or from public or private research centers.
L'archive ouverte pluridisciplinaire HAL, est destinée au dépôt et à la diffusion de documents scientifiques de niveau recherche, publiés ou non, émanant des établissements d'enseignement et de recherche français ou étrangers, des laboratoires publics ou privés. 

Not for reproduction, distribution or commercial use.

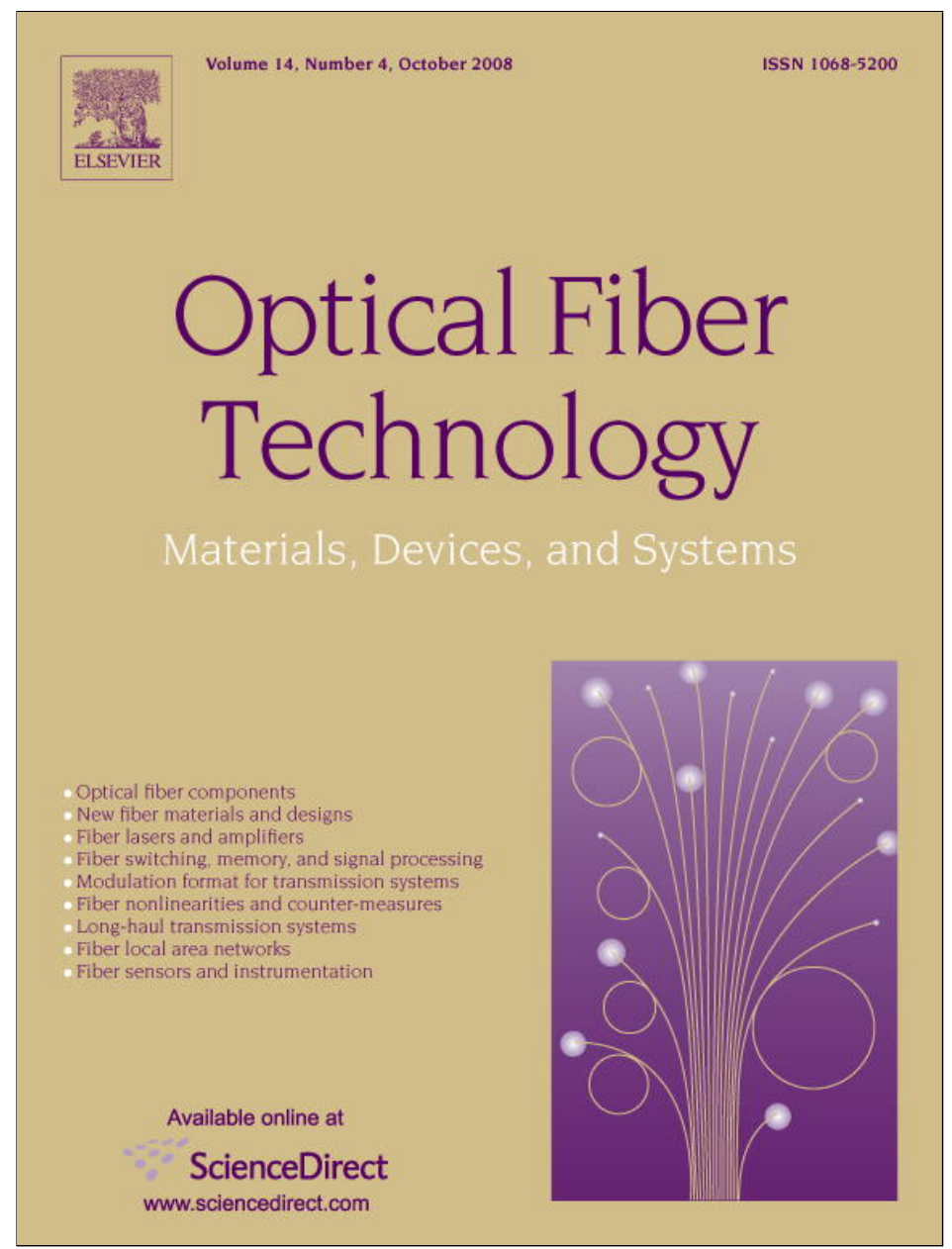

This article appeared in a journal published by Elsevier. The attached copy is furnished to the author for internal non-commercial research and education use, including for instruction at the authors institution and sharing with colleagues.

Other uses, including reproduction and distribution, or selling or licensing copies, or posting to personal, institutional or third party websites are prohibited.

In most cases authors are permitted to post their version of the article (e.g. in Word or Tex form) to their personal website or institutional repository. Authors requiring further information regarding Elsevier's archiving and manuscript policies are encouraged to visit:

http://www.elsevier.com/copyright 


\title{
Mechanism of multiple pulse formation in the normal dispersion regime of passively mode-locked fiber ring lasers
}

\author{
Adil Haboucha ${ }^{\text {a }}$, Andrey Komarov ${ }^{\mathrm{a}, 1}$, Hervé Leblond ${ }^{\mathrm{a}}$, François Sanchez ${ }^{\mathrm{a}, *}$, Gilles Martel ${ }^{\mathrm{b}}$ \\ a Laboratoire POMA UMR CNRS 6136, Université d'Angers, 2 Bd. Lavoisier, 49045 Angers Cedex 01, France \\ ${ }^{\mathrm{b}}$ Groupe d'Optique et d'Optronique, CORIA UMR CNRS 6614, Université de Rouen, 76801 Saint-Etienne du Rouvray Cedex, France
}

Received 12 March 2007; revised 18 September 2007

Available online 7 March 2008

\begin{abstract}
We identify the spectral gain filtering as a mechanism of multiple pulse formation in a fiber laser passively mode-locked through nonlinear polarization rotation operating in the normal dispersion regime. A correlation is theoretically established between the spectral gain bandwidth and the possibility for the laser to deliver several pulses by cavity round-trip: narrow spectrum favours multiple pulsing. Moreover the model allows to show that spectral loss or gain filtering are equivalent with respect to their consequences on the multiple pulsing regime. Experimental results obtained with an erbium-doped fiber laser and an ytterbium-doped fiber laser confirm the theoretical calculations. In addition, an indirect confirmation of the influence of the spectral filtering is proposed using an experimental setup involving a unique laser.

(c) 2008 Elsevier Inc. All rights reserved.
\end{abstract}

Keywords: Fiber laser; Passive mode-locking; Soliton; Multiple pulsing

\section{Introduction}

Multiple pulsing is a usual behaviour in passively modelocked fiber lasers, independently of the exact optical configuration. Indeed, it has been observed in figure eight lasers, stretched pulse lasers and in lasers passively mode-locked through nonlinear polarization rotation [1-6]. In the latter case, multiple pulsing occurs both in the normal and in the anomalous dispersion regime and also with different rare-earth ions such as erbium or ytterbium. In the anomalous dispersion regime, the quantization of the soliton energy is responsible for the generation of several pulses by cavity round-trip above some pump threshold value [6-9]. In the normal dispersion regime, the pulse energy is not quantified but multiple pulses are still obtained above some value of the pump power [10] or sometimes at the lasing threshold. In the following we will consider fiber lasers operating in the normal dispersion regime and passively mode-locked through nonlinear polarization rotation. In

\footnotetext{
* Corresponding author.

E-mail address: francois.sanchez@univ-angers.fr (F. Sanchez).

1 Permanent address: Institute of Automation and Electrometry, Russian Academy of Sciences, Akad. Koptyug Pr. 1, 630090 Novosibirsk, Russia.
}

our experiments, we have observed the following trends. Stable mode-locking with single pulse per cavity round-trip can be easily obtained with the ytterbium-doped double-clad fiber laser; multiple pulsing can be also found by a suitable adjustment of the intracavity phase plates [11-13]. In the case of the erbium-doped double-clad fiber laser, single-pulse modelocking is very difficult to obtain; the most usual behaviour is multiple pulsing. Single pulse per cavity round-trip occurs in a very narrow pumping range and can be reached only by first starting on a multiple pulsing state and then decreasing the pumping power near the lasing threshold. From the theoretical point of view, we have recently shown that additional spectrally selective losses are required to model several pulses by cavity round-trip in the normal dispersion regime [14]. This additional term is however difficult to justify because it is linked to uncontrolled wavelength-dependent losses. The aim of this paper is to clarify the influence of the spectral filtering on the formation of several pulses by cavity round-trip in the normal dispersion regime. The spectral gain bandwidth is intimately linked to the ability for the laser to deliver multiple pulsing. To demonstrate it we first consider in Section 2 theoretical results showing the effect of the spectral gain filtering on the laser operation of a passively mode-locked fiber laser exploiting 


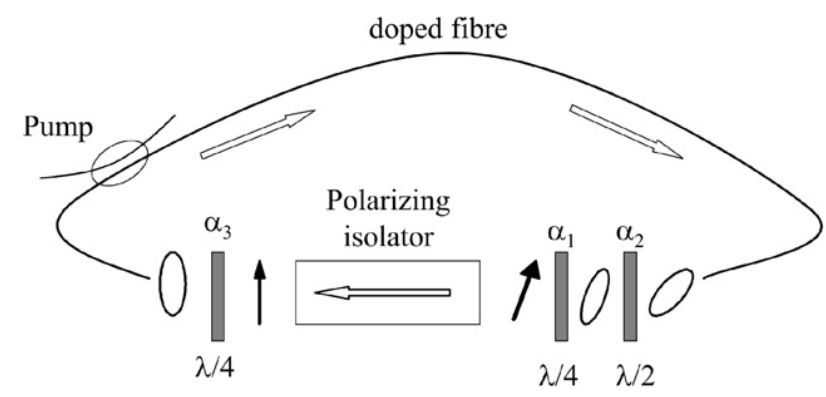

Fig. 1. Schematic representation of a fiber ring laser passively mode-locked through nonlinear polarization rotation.

the nonlinear polarization rotation. A first experimental confirmation of such behaviour is presented in Section 3 where we give typical results obtained with an erbium-doped and with an ytterbium-doped double-clad fiber lasers. Because it is difficult to have comparable experimental configurations for both lasers, we propose in Section 4 an original setup which would lead to fully convincing results. The corresponding theoretical results are given in the same section and permit to demonstrate that spectral loss filtering is equivalent to spectral gain filtering with respect to the multiple pulse formation.

\section{Theoretical model}

We consider a rare-earth-doped fiber ring laser passively mode-locked through nonlinear polarization rotation. The setup is schematically represented in Fig. 1. For isotropic fibers this scheme involves all necessary elements for control of nonlinear losses. After the polarizing isolator the electric field has a linear polarization. Such state of polarization does not experience polarization rotation in the fiber because the rotation angle is proportional to the area of the polarization ellipse. Consequently, it is necessary to place a quarter wave plate $3\left(\alpha_{3}\right.$ represents the orientation angle of one eigenaxis of the plate with respect to the laboratory frame). At the output of the fiber, the direction of the elliptical polarization of the central part of the pulse can be rotated toward the passing axis of the polarizer by the half wave plate 2 (the orientation angle is $\alpha_{2}$ ). Then this elliptical polarization can be transformed into a linear one by the quarter wave plate 1 (the orientation angle is $\alpha_{1}$ ). In this situation the losses for the central part of the pulse are minimum while the wings undergo strong losses. The setup of Fig. 1 has been modelled as follows [14]. The fiber has been assumed to have group velocity dispersion (GVD), optical Kerr nonlinearity and saturable gain. The nonlinear losses are described by solving in a first step the equations for a vectorial electric field propagating in a Kerr medium when dispersion is neglected, and then taking into account the three phase plates and the polarizer (self- and cross-phase modulation terms were considered together with four-wave mixing terms). On the other hand, a scalar equation has been written for a wave propagating in a saturable amplifying medium with GVD, to account for dispersion and gain. The resulting model assumes localized effect for the nonlinear loss due to the Kerr nonlinearity and the phase plates, while gain and GVD are distributed. In dimensionless form, the final set of equations for the electric field amplitude is [14]

$$
\begin{aligned}
& \frac{\partial E}{\partial \zeta}=\left(D_{\mathrm{r}}\right. \\
& \left.+i D_{i}\right) \frac{\partial^{2} E}{\partial \tau^{2}}+\left(G+i|E|^{2}\right) E \\
& E_{n+1}(\tau)=-\beta\left[\cos \left(p I_{n}+\alpha\right) \cos \left(\alpha_{1}-\alpha_{3}\right)\right. \\
& \left.+i \sin \left(p I_{n}+\alpha\right) \sin \left(\alpha_{1}+\alpha_{3}\right)\right] E_{n}(\tau),
\end{aligned}
$$

where $\zeta=z / L, \tau=t / \delta t, G=a /\left(1+b \int I d \tau\right), D_{\mathrm{r}}=G D_{\mathrm{r}}^{0}$ $+d_{\mathrm{r}}, D_{\mathrm{r}}^{0}=2 /\left(\left|\beta_{2}\right| L \omega_{\mathrm{g}}^{2}\right), \quad I_{n}=\left|E_{n}\right|^{2}, d_{\mathrm{r}}=2 \rho_{\mathrm{c}} /\left|\beta_{2}\right|, \quad p=$ $B \sin 2 \alpha_{3}, a=g_{0} L, b=I_{\mathrm{r}} \delta t /\left(P_{\mathrm{sat}} T_{\mathrm{a}}\right), \delta t=\sqrt{\left|\beta_{2}\right| L / 2}, I_{\mathrm{r}}=$ $1 / \gamma L, \alpha=2 \alpha_{2}-\alpha_{1}-\alpha_{3}$.

$D_{i}$ and $L(\mathrm{~m})$ are the dispersion and the length of the fiber, respectively $\left(D_{i}=-1\right.$ in the normal dispersion regime, and $D_{i}=1$ in the anomalous dispersion regime). $\gamma\left(\mathrm{W}^{-1} \mathrm{~m}^{-1}\right)$ is the nonlinear coefficient related to the nonlinear index coefficient $n_{2}$, and $B=1 / 3$ for silicate fibers [15]. $\beta$ is the transmission coefficient of the polarizer (free parameter). $\beta_{2}\left(\mathrm{ps}^{2} \mathrm{~m}^{-1}\right)$ is the second-order GVD. $T_{\mathrm{a}}=L n_{0} / c$ (s) is the photon round trip time, $n_{0}$ is the refractive index, $c$ is the velocity of light in free space, $g_{0}\left(\mathrm{~m}^{-1}\right)$ is the unsaturated gain, and $P_{\text {sat }}(\mathrm{W})$ is the saturating power. $P_{\text {sat }}=\left(h v \pi r^{2}\right) /\left(\sigma T_{1}\right)$, where $h v(\mathrm{~J})$ is the photon energy, $\sigma\left(\mathrm{m}^{2}\right)$ is the stimulated emission cross-section, $T_{1}$ (s) is the lifetime of the upper level of the lasing transition, and $r(\mathrm{~m})$ is the radius of the fiber core, $v$ is the optical frequency, and $h$ is the Planck constant. $\omega_{\mathrm{g}}(\mathrm{rad} / \mathrm{s})$ is the spectral gain bandwidth. $\rho_{\mathrm{c}}$ describes the frequency-dependent loss due to both additional spectrally selective elements for control of a radiation spectrum or uncontrolled spectrally selective losses related with intracavity elements. The parameter a represents the dimensionless pumping level.

The numerical procedure starts from the evaluation of the electric field after passing through the Kerr medium, the phase plates and the polarizer, using Eq. (2). The resulting electric field is then used as the input field to solve Eq. (1) over a distance $L$, using a standard split-step Fourier algorithm. The computed output field is used as the new input for Eq. (2). This iterative procedure is repeated until a steady-state is achieved.

In our previous works we showed that additional spectrally selective losses were necessary to achieve multiple pulsing in the normal dispersion regime [14]. Before to proceed, let us briefly recall some general features reported in [14]. It has been demonstrated that pulses appear one by one when the pump power is increased and that large hysteresis occurs versus the pumping parameter. Full detail, including the emergence of single and multiple pulsing is given in [14]. Although other parameters, such as pulse duration, are important to study, we focus in the following only on the number of pulses generated in the laser cavity. This is motivated by the fact that prior to optimize any pulse characteristics, it is needed to control the number of pulses by cavity round-trip.

For simplicity, we consider a single fiber operating in the normal dispersion regime at the lasing wavelength. Of course, the doping ion will be different depending on the exact operating wavelength but, in any case, the fiber core diameter is expected to allow a normal dispersion. In this section we consider that $\rho_{\mathrm{c}}=0$ (i.e., no additional spectrally selective losses) and vary the spectral gain bandwidth $\omega_{\mathrm{g}}$. For the numerical simulations we use the following parameters $\gamma=3 \times$ $10^{-3} \mathrm{~W}^{-1} \mathrm{~m}^{-1}, L=9 \mathrm{~m}, c=3 \times 10^{8} \mathrm{~ms}^{-1}, r=5 \times 10^{-6} \mathrm{~m}$, 


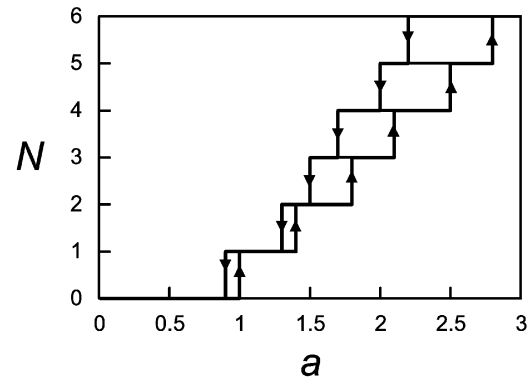

(a)

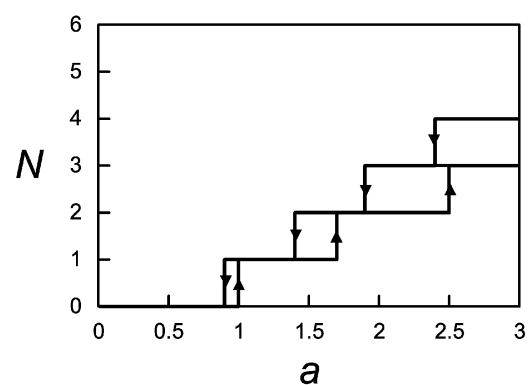

(b)

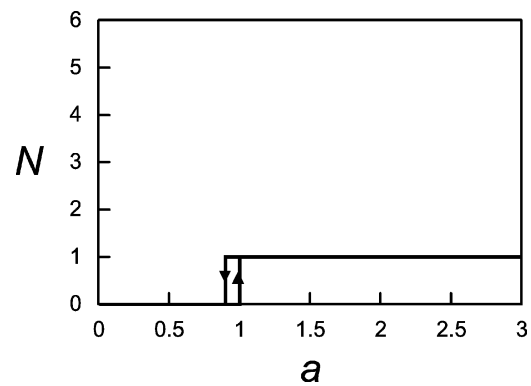

(c)

Fig. 2. Theoretical dependence of the number of pulses in the cavity versus the pumping parameter for different spectral gain bandwidths. The parameters used are $D_{i}=-1, \alpha=-1.1, \alpha_{1}=-0.2, \alpha_{3}=0.2, b=0.017$, and $\rho_{\mathrm{c}}=0$. (a) $\omega_{\mathrm{g}}=10^{12} \mathrm{rad} / \mathrm{s}$, (b) $\omega_{\mathrm{g}}=10^{13} \mathrm{rad} / \mathrm{s}$, and (c) $\omega_{\mathrm{g}}=10^{14} \mathrm{rad} / \mathrm{s}$.

$\sigma=2.5 \times 10^{-24} \mathrm{~m}^{2}, \beta_{2}=0.026 \mathrm{ps}^{2} \mathrm{~m}^{-1}, T_{1}=8 \times 10^{-4} \mathrm{~s}$, and $\beta=0.95$. Results of numerical simulations are summarized in Fig. 2 which gives the evolution of the number of pulses in the cavity as a function of the pumping parameter a for 3 different values of $\omega_{\mathrm{g}}$. While in our previous papers we presented such evolutions in presence of spectrally selective losses leading to results similar to those of Fig. 2a, it is the gain which limits the effective optical bandwidth in this study. Fig. 2 a proves that additional spectrally selective losses are not required to model multiple pulsing operation. Indeed, the natural spectral selection of the gain also leads to several pulses by cavity round-trip. Inspection of plots of Fig. 2 reveals that, for a given pumping parameter, the multiple pulsing behaviour tends to disappear when the spectral gain bandwidth is increased. The pump power for which a second pulse appears is pushed toward higher values. These results are very important from a practical point of view because they mean that the spectroscopic properties of the amplifying medium are mainly responsible for the emergence of several pulses in the normal dispersion regime.

However, multiple-pulsing behaviour can be more or less favoured by the orientation of the intra-cavity phase plates. For the same laser parameters as in Figs. $2 a$ and $2 b$, but changing the phase plates orientations, additional simulations show that the single pulse operation occurs for pumping rates up to 2 . The conditions favourable for the single pulse operation are the following: the rotation angle of polarization ellipse for central part of the pulse must be less than the optimal one. This is achieved by changing the orientation angle of the half wave plate and by decreasing the orientation angle for the quarter wave plate $\alpha_{3}$. In this case the polarization entering into the fiber is close to linear and the rotation angle velocity becomes small (i.e., the effective rotation is small). As a consequence, the most powerful pulse undergoes less loss and wins competition. In the case of anisotropic fiber this mechanism allowing to suppress multiple pulsing disappears. Indeed, the fiber anisotropy works as an additional phase plate and transforms the linear polarization into an elliptic one. It is probably this circumstance which prevents to obtain the single pulse operation in experiments for narrow spectral gain bandwidth.

The important result in our theoretical analysis is that, for large values of $\omega_{\mathrm{g}}$, multiple pulsing is not possible whatever the orientation of the phase plates. On the other hand, for narrow spectral gain bandwidth, multiple pulsing is the most probable behaviour although for moderate pumping rates, single pulse regime can be obtained with a suitable adjustment of the phase plates. However, the range of values of the $\alpha_{i}$ 's for which a single pulse operation is possible decreases while the gain bandwidth decreases. This means that it is more difficult to obtain the single pulse regime. We expect that this range tends to vanish for anisotropic fibers. This point cannot be investigated in the framework of our model. In summary, spectral gain filtering is a necessary condition for multiple pulse operation. Consequently, it appears as a mechanism of the formation of several pulses per cavity round-trip.

However, in Ref. [16], a strong additional spectral filtering $(10 \mathrm{~nm})$ did not lead to multiple pulsing (in [16], it is used a spectral loss filtering while we have considered the natural spectral gain filtering: it will be shown in the last paragraph that both filtering are equivalent). In fact, a careful reading of the cited paper allows to rule out any contradiction. Notice first that experimental results do not formally allow to conclude if there are several pulses separated by some tens of picoseconds. Indeed, when pulses are not bound, the optical spectrum is not modulated; therefore an absence of modulation does not imply a single pulse by cavity round-trip. More important is the fact that the pumping power is experimentally limited to $20 \%$ above the mode-locked threshold. Such pumping ratio is probably too small to generate several pulses by cavity round-trip as shown in Fig. 2a. Hence, the results of Ref. [16] are compatible with our predictions.

\section{Experimental results}

The aim of this section is to present two experimental examples which confirm the theoretical predictions. Because it is not possible to vary the spectral gain bandwidth of the amplifying medium, we consider the case of the erbium-doped fiber for the narrowest bandwidth (about $30 \mathrm{~nm}$ ) and the case 


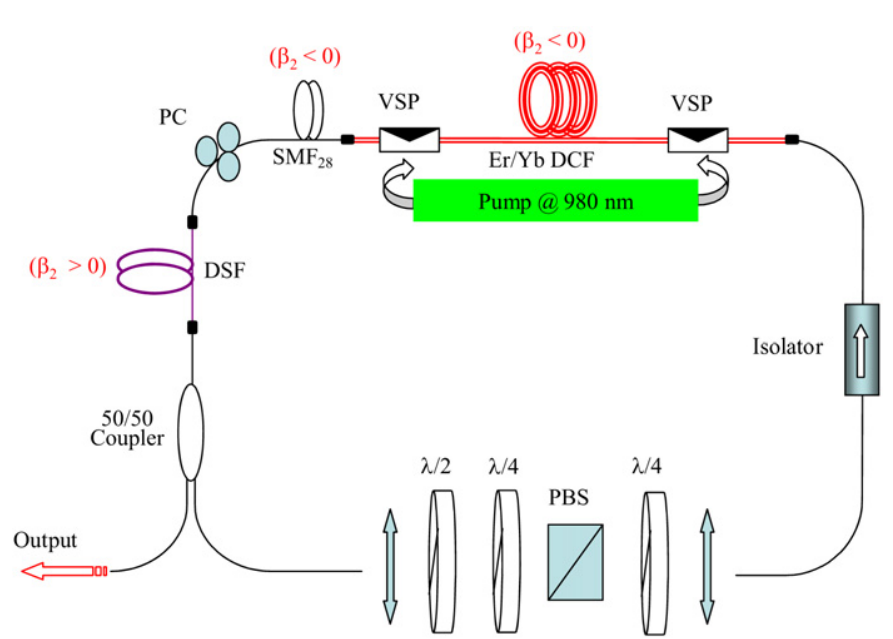

Fig. 3. Experimental setup for the erbium-doped fiber laser. DSF: dispersion shifted fiber, PC: polarization controller, VSP: v-groove side pumped, DCF: double-clad fiber, PBS: polarization beam splitter.

of the ytterbium-doped fiber for the larger bandwidth (about $60 \mathrm{~nm})$.

Let us first consider the case of the erbium fiber which is in fact an erbium/ytterbium-doped fiber, in which the ytterbium ions are optically pumped at $980 \mathrm{~nm}$ and transfer their energy to the erbium ions thus creating gain at $1.55 \mu \mathrm{m}$. The experiments have been performed with an erbium-ytterbium-doped doubleclad fiber amplifier in a unidirectional ring cavity as shown in Fig. 3. The double-clad fiber consists in a single-mode core doped with ytterbium (for the pumping) and erbium ions (emitting at $1.55 \mu \mathrm{m}$ ) and two clads. The inner cladding allows having the multimode propagation of the pump beam thus allowing to use compact high power semiconductor lasers for the optical pumping. It has a flower shape in order to break the cylindrical symmetry and then to increase the pump absorption coefficient. Two semiconductor laser diodes in counter-propagating configuration are used. Both have an available output power of about $3.5 \mathrm{~W}$. The V-groove technique is employed to launch the light into the fiber [17]. This technique allows to leave the fiber ends free and is then well adapted for ring cavities [11]. An independent-polarization optical isolator is used to obtain unidirectional oscillation. The double-clad fiber (DCF) and the standard SMF fiber have anomalous dispersion $\left(\beta_{2}<0\right)$. In order to manage the total cavity dispersion, a piece of dispersion shifted fiber is used (DSF, $\beta_{2}>0$ ). The different lengths are such that the laser operates in the stretched pulse regime where the total cavity dispersion is slightly positive $\beta_{2} L=0.06 \mathrm{ps}^{2}$, the repetition rate is about $8.85 \mathrm{MHz}$. The mode-locking is achieved through nonlinear polarization rotation occurring in the fibers and the resulting fast intensity-dependent losses produced by the intracavity polarization beam splitter. Three phase plates are also used in order to change or to optimize the operating regime of the laser. Indeed, a simple rotation of a phase plate allows to observe different regimes such as continuous, $Q$-switch, regular or irregular mode-locking, multiple pulsing, etc. as already reported both experimentally with a Yb-doped fiber laser [11] and theoretically [18]. A 50/50 single-mode fiber coupler is used to extract the energy from the cavity. The

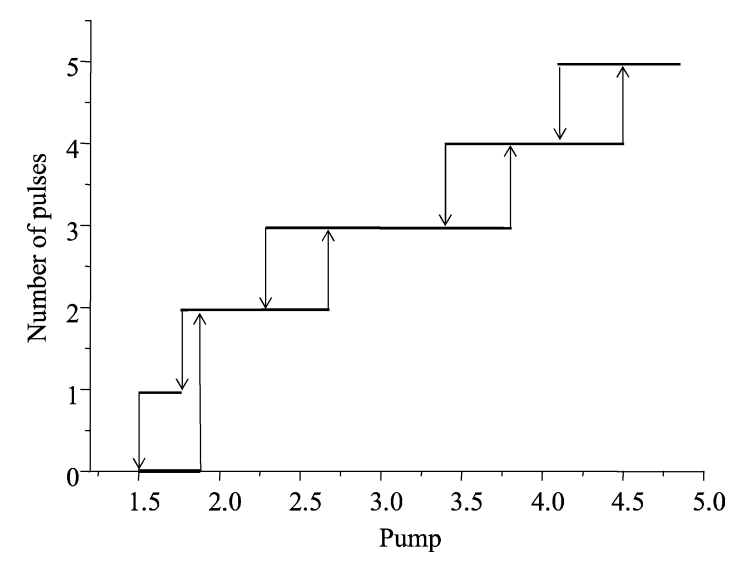

Fig. 4. Experimental results for the erbium-doped fiber laser. Evolution of the number of pulses versus pump power.

output is analyzed with a $12 \mathrm{GHz}$ photodetector and a fast 12 $\mathrm{GHz}$ oscilloscope. An optical spectrum analyzer and an optical autocorrelator are also used when needed.

The procedure of investigation of the multiple pulsing is the following. The pump power is fixed to an intermediate value and the different phase plates and polarization controllers are adjusted to obtain a regular signal consisting in several pulses per cavity round-trip. The bunch of pulses is well resolved with the fast photodetector and the high bandwidth oscilloscope (pulses are typically separated by some $10 \mathrm{ps}$ ). Therefore, it is possible to count the number of pulses for increasing and decreasing pumping power. Experimental results are summarized in Fig. 4. When the pump power increases, the laser directly starts in the regime of 2 pulses per cavity round-trip. When the pumping is further increased, additional pulses appear one by one, up to 5. At this pumping level, if the power decreases, pulses disappear also one by one but for different values of switching powers. Results clearly demonstrate pump power hysteresis. Similar results can be obtained in the anomalous dispersion regime. We have tried many positions for the phase plates and also several configurations with different values of the GVD. We never observed spontaneous single pulse operation in a wide range of pumping rates (i.e., the laser cannot operate directly with a single pulse, this regime is obtained only in a small range of pumping and after an evolution in a multiple pulse state). The evolution of the number of pulses can be different from the one of Fig. 4 but multiple pulsing always occurred. The spectral gain bandwidth is of the order of $\omega_{\mathrm{g}} \approx 2 \times 10^{13} \mathrm{rad} / \mathrm{s}$ and experimental results favourably compare with theoretical evolution of Fig. 2b. What is important in this comparison is not the exact evolution neither the possible differences in the hysteresis curves (it is possible to obtain very different evolutions with a simple rotation of a phase plate) but rather the fact that multiple pulsing occurs experimentally for any position of the phase plates.

Let us now consider the case of the ytterbium-doped doubleclad fiber lasers, using already published experimental results. The spectral gain bandwidth is of the order of $\omega_{\mathrm{g}} \approx 10^{14} \mathrm{rad} / \mathrm{s}$ and we can expect that single pulse operation is easily achievable (see Fig. 2c). The experimental setup is similar to the one used with the erbium-doped fiber and the mode-locking is ob- 
tained through nonlinear polarization rotation. As it is predicted by the theory, the laser generally delivers one pulse per cavity round-trip [12]. However, as in previously published numerical simulations [18] (not reported here because they are related to $Q$-switching and unstable regimes), other operating regimes with several pulses can be experimentally obtained by a suitable adjustment of the phase plates [11]. Recall that the operating regime of the laser strongly depends on the orientation of the phase plates $[11,19]$.

In summary, the experimental results, obtained in the normal dispersion regime, lead to the following comments. Because of its narrow spectral gain bandwidth, the erbium-doped fiber laser operates always in a multiple pulsing regime. Single pulse operation can be obtained only in a narrow pumping range and is reached by first starting on two-pulse regime and then decreasing the pumping power (in other words, single pulse is not a self-starting regime). As mentioned in the theoretical section, the residual birefringence of the fibers could participate to a significant reduction of the range of parameters allowing a single-pulse operation. In contrast, the ytterbium laser easily delivers a single pulse by cavity round-trip even if other behaviours can be found both theoretically [18] and experimentally [11] (CW, $Q$-switch, imperfect mode-locked). The case of the erbium is particular because other teams have observed a regular and spontaneous single pulse regime. It is well known that co-doping [20] and also high erbium concentration [21,22] can significantly modify the spectral properties and, in particular, the gain bandwidth. This fact explains why an erbium-doped fiber laser passively mode-locked can operate on single pulse or multiple pulse regime depending on the exact composition of the doped fiber.

\section{Proposal for a new experimental confirmation}

Although previous experimental results confirm the theoretical predictions, they are not completely satisfactory because they are obtained with two distinct lasers. This problem cannot be overcome because it is not possible to vary the spectral gain bandwidth in a unique laser. The ideal will be a fiber laser with a sufficiently large spectral gain bandwidth allowing a single pulse operation and then to reduce its bandwidth in order to highlight the generation of more and more pulses. Unfortunately this theoretical experiment cannot be realized. However, there is an indirect way which will consist to start with a fiber laser having a large spectral gain bandwidth and to insert in the cavity a spatially dispersive element in order to be able to filter the spatially spread spectrum. When the filter is adjusted to reduce the selected spectrum we can expect that the laser becomes multiple pulsing. Of course, this experiment does not allow varying the gain bandwidth but it allows to control the spectral loss filtering. We have not at this time all the necessary material to conduct such experiments. We can however investigate theoretically the influence of a reduction of the spectral loss bandwidth in order to check if it is equivalent to a reduction of the gain bandwidth. For that, we solve numerically Eqs. (1) and (2) where the spectral gain bandwidth is fixed to $\omega_{\mathrm{g}}=10^{14} \mathrm{rad} / \mathrm{s}$ and take different values for the spectral

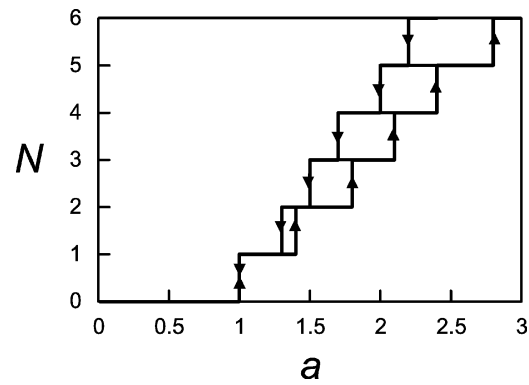

(a)

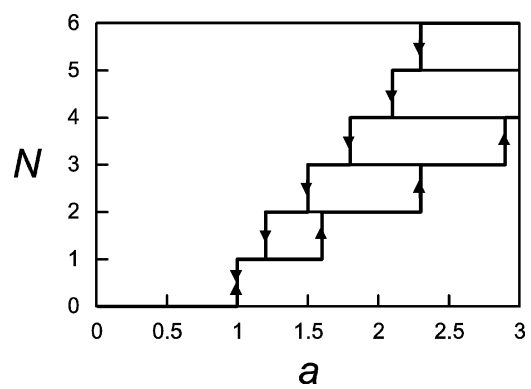

(b)

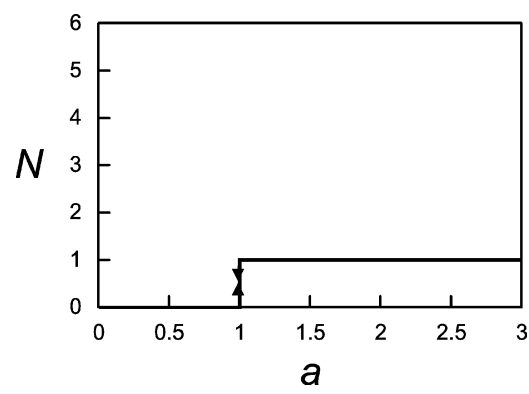

(c)

Fig. 5. Theoretical dependence of the number of pulses in the cavity versus the pumping parameter for different spectral loss bandwidths. The parameters used are $D_{i}=-1, \alpha=-1.1, \alpha_{1}=-0.2, \alpha_{3}=0.2$, and $\omega_{\mathrm{g}}=10^{14} \mathrm{rad} / \mathrm{s}$. (a) $\omega_{\mathrm{d}}=10^{12} \mathrm{rad} / \mathrm{s}$, (b) $\omega_{\mathrm{d}}=10^{13} \mathrm{rad} / \mathrm{s}$, and (c) $\omega_{\mathrm{d}}=10^{14} \mathrm{rad} / \mathrm{s}$.

loss bandwidth $\left(\omega_{\mathrm{d}}=1 / \sqrt{\rho_{\mathrm{c}} L}\right)$. Results are presented in Fig. 5 which gives the evolution of the number of pulses for increasing and decreasing pumping parameter. The parameters used are exactly the same as those taken in Section 2 except that the spectral gain bandwidth is fixed. It is clearly demonstrated that the spectral loss filtering induces multiple pulses regime. When the spectral loss bandwidth is equal to the spectral gain bandwidth, the laser operates exclusively in a single pulse regime as shown in Fig. 5c and multiple pulsing is not possible. We have checked by running simulations for values of the pumping parameter $a$ up to 4 that the laser remains in single pulse regime. Hence multiple pulse operation appears to be suppressed and not only shifted to higher values of $a$. Higher values will not be of practical interest because they will correspond to pumping powers above 4 times the pump power threshold. While the pump power is increased, the pulse energy increases.

While the spectral loss bandwidth is decreased, the laser exhibits several pulses by cavity round-trip (see Figs. 5a and 5b). These theoretical results demonstrate that spectral loss or gain filtering are equivalent with respect to their influence on the laser ability to deliver multiple pulses per cavity round-trip. In- 
deed, without spectral filtering (gain or loss), multiple pulsing is not possible in the mode-locked regime and the laser delivers one pulse by cavity round-trip. When spectral filtering acts, the laser can operate on multiple-pulse regime although singlepulse is not completely forbidden. The latter is however more difficult to obtain.

\section{Conclusion}

In this paper we have identified the spectral gain filtering as a mechanism of multiple pulse formation in a passively mode-locked fiber laser. The work was motivated by the fact that, in the normal dispersion regime, the ytterbium-doped fiber laser generally operates with a single pulse per cavity roundtrip while this regime is very difficult to obtain with our own erbium-doped fiber laser. The main difference between the two doping ions is the resulting spectral gain bandwidth. We have theoretically demonstrated that multiple pulsing is not possible if there is not enough spectral gain filtering. This is in good agreement with the experimental data because the spectral gain bandwidth is larger in the case of the ytterbium-doped fiber. These results make a direct link between the exact composition of the doped fiber and the operating regime in passively mode-locked experiments because even for a given doping ion, co-doping or high doping concentration significantly affects the spectral properties of the doped material. In order to confirm the theoretical results with a single laser, which would be more convincing, we have proposed a new experiment in which a spectrally selective element with a variable bandwidth is inserted inside the cavity. Of course, such experiment does not allow varying the spectral gain bandwidth but rather the spectral bandwidth of the losses. We have then theoretically checked that spectral loss and gain filtering are equivalent because both lead to the generation of multiple pulses by cavity round-trip when their bandwidth is narrow enough.

\section{Acknowledgment}

This research was supported by a Marie Curie International Fellowship within the 6th European Community Framework Programme.

\section{References}

[1] M.J. Guy, D.U. Noske, J.R. Taylor, Generation of femtosecond soliton pulses by passively mode locking of an ytterbium-erbium figure-of-eight fibre laser, Opt. Lett. 18 (1993) 1447-1449.

[2] D.J. Richardson, R.I. Laming, D.N. Payne, V.J. Matsas, M.W. Phillips, Pulse repetition rates in passive, self-starting, femtosecond soliton fibre laser, Electron. Lett. 27 (1991) 1451-1453.
[3] V.J. Matsas, D.J. Richardson, T.P. Newson, D.N. Payne, Characterization of a self-starting, passively mode-locked fiber ring laser that exploits nonlinear polarization rotation, Opt. Lett. 18 (1993) 358-360.

[4] M.J. Guy, D.U. Noske, A. Boskovic, J.R. Taylor, Femtosecond soliton generation in praseodymium fluoride fiber laser, Opt. Lett. 19 (1994) 828830 .

[5] K.S. Abedin, J.T. Gopinath, L.A. Jiang, M.E. Grein, H.A. Haus, E.P. Ippen, Self-stabilized passive, harmonically mode-locked stretched-pulse erbium fiber ring laser, Opt. Lett. 27 (2002) 1758-1760.

[6] D.Y. Tang, W.S. Man, H.Y. Tam, Stimulated soliton pulse formation and its mechanism in a passively mode-locked fibre soliton laser, Opt. Commun. 165 (1999) 189-194.

[7] B. Zhao, D.Y. Tang, P. Shum, Y.D. Gong, C. Lu, W.S. Man, H.Y. Tam, Energy quantization of twin-pulse solitons in passively mode-locked fiber ring lasers, Appl. Phys. B 77 (2003) 585-588.

[8] A.B. Grudinin, D.J. Richardson, D.N. Payne, Energy quantization in figure eight fibre laser, Electron. Lett. 28 (1992) 67-68.

[9] D.Y. Tang, L.M. Zhao, B. Zhao, A.Q. Liu, Mechanism of multisoliton formation and soliton energy quantization in passively mode-locked fiber lasers, Phys. Rev. A 72 (2005) 043816.

[10] A. Haboucha, A. Komarov, H. Leblond, F. Sanchez, Multiple pulsing and hysteresis phenomena in fiber lasers: theory and experiment, in: Proceedings of International Conference on Transparent Optical Networks, ICTON 2006, vol. 1, Nottingham, UK, June 2006, pp.187-190.

[11] A. Hideur, T. Chartier, M. Sahli, C. Özkul, M. Brunel, F. Sanchez, Modelock, $Q$-switch and $\mathrm{CW}$ operation of an Yb-doped double-clad fiber ring laser, Opt. Commun. 198 (2001) 141-146.

[12] A. Hideur, T. Chartier, M. Brunel, S. Louis, C. Özkul, F. Sanchez, Generation of high energy femtosecond pulses from a side-pumped $\mathrm{Yb}$-doped double-clad fiber laser, Appl. Phys. Lett. 79 (2001) 3389-3391.

[13] H. Leblond, M. Salhi, A. Hideur, T. Chartier, M. Brunel, F. Sanchez, Experimental and theoretical study of the passively mode-locked ytterbiumdoped double-clad fiber laser, Phys. Rev. A 65 (2002) 063811.

[14] A. Komarov, H. Leblond, F. Sanchez, Multistability and hysteresis phenomena in passively mode-locked fibre lasers, Phys. Rev. A 71 (2005) 053809.

[15] G.P. Agrawal, Nonlinear Fiber Optics, second ed., Academic Press, New York, 1995.

[16] A. Chong, J. Buckley, W. Renninger, F. Wise, All-normal dispersion femtosecond fiber laser, Opt. Express 14 (2006) 10095-10100.

[17] D.J. Ripin, L. Goldberg, High efficiency side-coupling of light into optical fibers using imbedded v-grooves, Electron. Lett. 31 (1995) 22042205.

[18] A. Komarov, H. Leblond, F. Sanchez, Theoretical analysis of the operating regime of a passively mode-locked fiber laser through nonlinear polarization rotation, Phys. Rev. A 72 (2005) 063811(7).

[19] B. Ortaç, A. Hideur, M. Brunel, T. Chartier, M. Salhi, H. Leblond, F. Sanchez, Characterization of an ytterbium-doped double-clad fiber laser passively mode-locked by nonlinear polarization rotation, Appl. Phys. B 77 (2003) 589-594.

[20] J.L. Wagener, P.F. Wysocki, M.J.F. Digonnet, H.J. Shaw, Effects of concentration and clusters in erbium-doped fiber lasers, Opt. Lett. 18 (1993) 2014-2016.

[21] B.N. Samson, W.H. Loh, J.P. de Sandro, Experimental evidence of differences in the absorption spectra of clustered and isolated ions in erbiumdoped fibers, Opt. Lett. 22 (1997) 1763-1765.

[22] S. Colin, E. Contesse, P. LeBoudec, G. Stephan, F. Sanchez, Evidence of saturable absorption effect in heavily erbium-doped fibers, Opt. Lett. 21 (1996) 1987-1989. 